\section{Intestinal obstruction in an adult with Down's syndrome}

\author{
Altaf K Awan FRCS ${ }^{1}$ David A L Macafee MRCS $^{1}$ \\ Richard I Hall MD FRCS ${ }^{2}$
}

J R Soc Med 2004;97:334-335

In an adult with Down's syndrome, intestinal obstruction may be due to a developmental malformation.

\section{CASE HISTORY}

A man with Down's syndrome, age 48, was admitted after 24 hours of vague abdominal pain and vomiting. A similar episode 3 years previously had resolved spontaneously and he had been discharged without special investigations. The abdomen was distended and mildly tender with highpitched bowel sounds. X-rays revealed dilated small and large bowel loops but the anatomical distribution of the bowel loops, which was abnormal, was not considered relevant at this point (Figure 1). The provisional diagnosis was intestinal pseudo-obstruction and conservative management was initiated. However, the retching and vomiting continued and an unprepared barium enema then showed a tortuous large bowel with an obstruction at the level of the proximal ascending colon (Figure 2). At laparotomy, he was found to have a midgut malrotation with a volvulus of the small bowel. The caecum and ascending colon were mobile and redundant, with caecum and part of ascending colon lying to the left of the midline. The hepatic flexure, transverse colon and left colon were in their normal anatomical positions, but a peritoneal band lay across the ascending colon and reached the lateral abdominal wall. This band had caused a stricture in the underlying colon and the caecum had become ischaemic. The small bowel was dusky but recovered immediately after counter-clockwise derotation. The band across the ascending colon was divided and the gut was restored to a normal anatomical position after a limited hemicolectomy to remove the area of stricture and ischaemic-looking caecum. The patient recovered without incident. Postoperatively the parents reported that their son had suffered from intermittent retching and bilious vomiting for several years. Macroscopically, the right colon was dilated $(8 \mathrm{~cm})$; microscopically there was attenuation of

'Division of Gastrointestinal Surgery, Queen's Medical Centre, Nottingham; ${ }^{2}$ Derby City General Hospital, Derby, UK

Correspondence to: A K Awan, Division of GI Surgery, E Floor, West Block,

E-mail: altaf.awan@nottingham.ac.uk

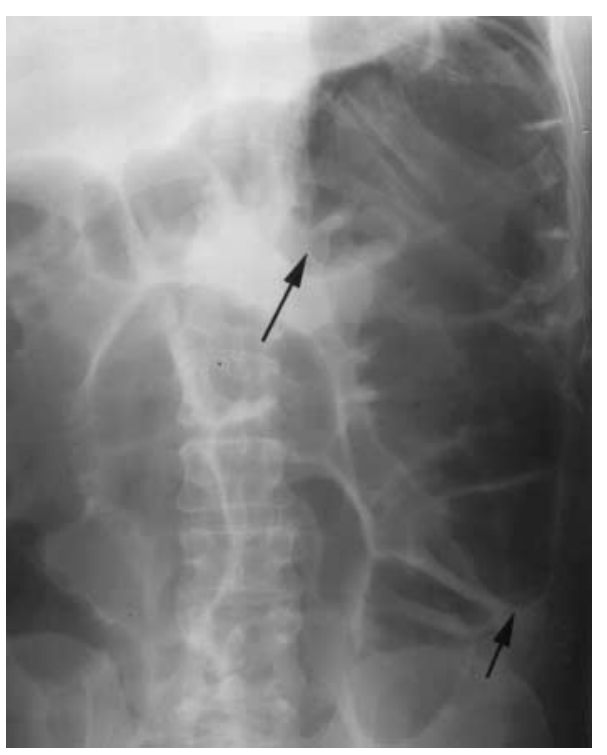

Figure 1 Preoperative abdominal X-ray showing a dilated caecum lying to the left of the midline (arrows)

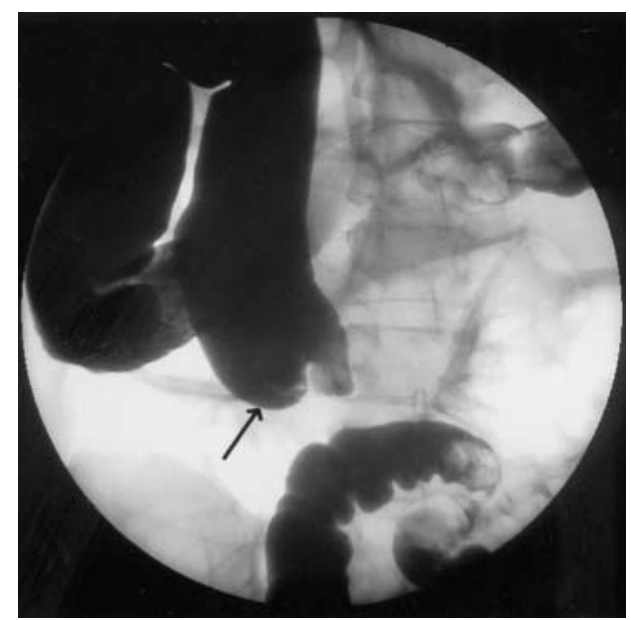

Figure 2 Preoperative barium enema. Arrow points to area where peritoneal band lay across the ascending colon, resulting in large-bowel obstruction

the wall and thinning of the mucosa but no evidence of ischaemia or malignancy.

\section{COMMENT}

The incidence of Down's syndrome in England and Wales is between one and two per 1000 births. ${ }^{1}$ Infants with Down's syndrome are at twenty times excess risk of having gastrointestinal abnormalities, ${ }^{2}$ and one of these, malrotation of the midgut, commonly presents as intestinal obstruction in neonatal life. ${ }^{3}$ This complication is seldom encountered in adults, ${ }^{4}$ but it may become more frequent with the greater survival of individuals with Down's syndrome into adult life ${ }^{5}$ (in Western countries mean survival now exceeds 50 years $\left.^{6}\right)$. 
Most of the malrotations that are recognized are causing no symptoms and are diagnosed incidentally on radiography. ${ }^{7}$ The risk of this malformation is that it predisposes to a midgut volvulus due to a narrow mesenteric fixation. Patients can present acutely, with intestinal obstruction and ischaemia, or may experience years of vague abdominal pain and recurrent attacks of retching or vomiting. ${ }^{8}$

Contrast studies may reveal duodenal obstruction, duodenojejunal flexure located to the right of the spine and small bowel confined to the right side of abdomen, ${ }^{4}$ but a normal small-bowel study does not exclude the possibility of malrotation ${ }^{7}$ and barium enema may be necessary. Computed tomography may detect a midgut malrotation, with or without volvulus, by demonstrating spiralling of the mesentery around the superior mesenteric artery and the superior mesenteric vein lying to the left of the artery. ${ }^{9,10}$ This vascular anatomy may also be evident on duplex ultrasound. ${ }^{11}$ Surgical treatment entails counter-clockwise untwisting of the volvulus and careful assessment of bowel viability. Any abnormal bands should be divided (Ladd's procedure). Normal anatomy of the intestinal tract can be restored by duodenopexy and suture fixation of the caecum and ascending colon to the right abdominal wall and retroperitoneum. ${ }^{4}$

\section{REFERENCES}

1 Huang T, Watt $\mathrm{H}$, Wald $\mathrm{N}$, et al. Birth prevalence of Down's syndrome in England and Wales 1990 to 1997. J Med Screen 1998;5:213-14

2 Torfs CP, Christianson RE. Anomalies in Down syndrome individuals in a large population-based registry. Am J Med Genet 1998;77:431-8

3 Devlin HB, Williams RS, Pierce JW. Presentation of midgut malrotation in adults. BMJ 1968;i:803-7

4 Dietz DW, Walsh RM, Grundfest-Broniatowski S, Lavery JC, Fazio VW, Vogt DP. Intestinal malrotation: a rare but important cause of bowel obstruction in adults. Dis Colon Rectum 2002;45: 1381-6

5 Hijii T, Fukushige J, Igarashi H, Takahashi N, Ueda K. Life expectancy and social adaptation in individuals with Down syndrome with and without surgery for congenital heart disease. Clin Pediatr (Phila) 1997; 36:327-32

6 Janicki MP, Dalton AJ, Henderson CM, Davidson PW. Mortality and morbidity among older adults with intellectual disability: health services considerations. Disabil Rehabil 1999;21(5):284-94

7 Balthazar EJ. Intestinal malrotation in adults. Roentgenographic assessment with emphasis on isolated complete and partial nonrotations. AJR Am J Roentgenol 1976;126:358-67

8 von Flue M, Herzog U, Ackermann C, Tondelli P, Harder F. Acute and chronic presentation of intestinal nonrotation in adults. Dis Colon Rectum 1994;37:192-8

9 Paul AB, Dean DM. Computed tomography in volvulus of the midgut. Br J Radiol 1990;63:893-4

10 Nichols DM, Li DK. Superior mesenteric vein rotation: a CT sign of midgut malrotation. AJR Am J Roentgenol 1983;141:707-8

11 Gaines PA, Saunders AJ, Drake D. Midgut malrotation diagnosed by ultrasound. Clin Radiol 1987;38:51-3

\section{Cytomegalovirus infection in Crohn's colitis}

\author{
S Olsen MB MRCS J Gilbert MS FRCS
}

J R Soc Med 2004;97:335-336

CLINICAL SECTION, 16 JANUARY 2003

Cytomegalovirus infection can complicate Crohn's colitis. Early detection and diagnosis is important in relation to treatment.

\section{CASE HISTORY}

A man of 70 was seen at the colorectal outpatient clinic after a month of diarrhoea, weight loss, anaemia and rectal bleeding. He was admitted, and on flexible sigmoidoscopy he was found to have extensive severe colitis with ulceration and pseudopolyposis but rectal sparing. Microscopy revealed deep fissuring ulcers and inflammatory infiltrate but no cryptitis, crypt abscesses or granulomas. There was no evidence of superimposed infection, dysplasia or malignancy. Crohn's colitis was diagnosed and he responded well to intravenous hydrocortisone $100 \mathrm{mg}$ three times daily and mesalazine $400 \mathrm{mg}$ three times daily. He was admitted subsequently with a relapse which responded to steroid therapy.

Six weeks after his second discharge he attended the accident and emergency department because of diarrhoea, anaemia and weight loss. Admitted under the care of the medical team he was treated for two weeks with oral prednisolone $30 \mathrm{mg}$ daily and mesalazine. Clinically he deteriorated, with a rise in C-reactive protein and a fall in haemoglobin, white blood cell count and albumin. On day 14 he was reviewed by the gastroenterology team and the treatment was changed to intravenous hydrocortisone $100 \mathrm{mg}$ three times daily; the mesalazine was stopped. On day 16 the colorectal team took over his care. Despite high-dose immunosuppressive treatment he continued to deteriorate and was transferred to the high dependency unit for correction of refractory hypokalaemia. On day 24, colonoscopy revealed severe active colitis to the ascending colon; multiple biopsies were taken. 3 days later, because of further clinical deterioration, he underwent subtotal colectomy with end ileostomy and cutaneous mucous fistula of the rectum. On the day after the operation, a report on the colonoscopic biopsies became available. As

Department of General Surgery, Wexham Park Hospital, Slough SL2 4HI, UK Correspondence to: Miss Sisse Olsen, Department of Surgical Oncology and Technology, 10th Floor OEQM Wing, St Mary's Hospital, London W2 1NY, UK E-mail: s.olsen@ic.ac.uk 